\title{
Patient and Healthcare Professionals Preference for Brenzys vs. Enbrel Autoinjector for Rheumatoid Arthritis: A Randomized Crossover Simulated-Use Study
}

\author{
Marc Egeth (1) - Jennifer Soosaar - Peter Nash $\cdot$ Denis Choquette \\ Ricardo Infante $\cdot$ Dena Rosen Ramey $\cdot$ Sevag Sahakian · \\ Angela Lai $\cdot$ Jin Ju Kim $\cdot$ David Wu
}

Received: February 3, 2017 / Published online: April 17, 2017

(c) The Author(s) 2017. This article is an open access publication

\section{ABSTRACT}

Introduction: Brenzys was developed as an etanercept biosimilar of Enbrel. The aim of this study was to assess preference and perceived ease of use for the new Brenzys autoinjector compared to the currently available marketed Enbrel MYCLIC autoinjector (Australia) and

Enhanced content To view enhanced content for this article go to http://www.medengine.com/Redeem/ F708F0604C53C926.

Electronic supplementary material The online version of this article (doi:10.1007/s12325-017-0523-x) contains supplementary material, which is available to authorized users.

M. Egeth $(\bowtie) \cdot J$. Soosaar

Core Human Factors, Inc., Bala Cynwyd, PA, USA

e-mail: egeth@corehf.com

P. Nash

Dept of Medicine, University of Queensland,

Sunshine Coast, QLD, Australia

D. Choquette

Institut de recherche en rhumatologie de Montréal, CHUM ( Notre-Dame), Université de Montréal,

Montréal, Canada

R. Infante

Medical Affairs, Merck \& Co., Inc., Kenilworth, NJ,

USA

D. R. Ramey

Center for Observational \& Real World Evidence,

Merck \& Co., Inc., Kenilworth, NJ, USA
Enbrel SureClick autoinjector (Canada) for the treatment of rheumatoid arthritis (RA). Because RA affects manual dexterity, ease of use of an autoinjector is a particularly important consideration in developing effective self-delivery of long-term courses of therapy.

Methods: Patients $(N=191)$ reporting a diagnosis of RA and nurses and rheumatologists $(N=90)$ with experience managing RA were shown how to use Brenzys and Enbrel autoinjectors (in counterbalanced order between participants), then they used each autoinjector by injecting into a pad simulating skin, and completed a questionnaire. Study sessions took place in Australia and Canada.

\section{S. Sahakian}

Medical Affairs, Merck Canada Inc., Kirkland,

Québec, Canada

\section{A. Lai}

Medical Affairs, MSD Australia, Macquarie Park,

NSW, Australia

\section{J. J. Kim}

MSD Korea Ltd., Mapo-GU, Seoul, Korea

D. $\mathrm{Wu}$

Merck \& Co., Inc., Kenilworth, NJ, USA 
Results: A binomial test showed that significantly more patients indicated that the Brenzys autoinjector was easier to use than the Enbrel autoinjector (79\% reporting Brenzys easier to use; $p<0.001$, two-sided, 95\% CI [73\%, 85\%]). In addition, significantly more nurses and rheumatologists with experience managing RA also indicated that the Brenzys autoinjector was easier to use (86\%; $p<0.001$, two-sided, 95\% CI $[77 \%, 92 \%)$ and that they would recommend the buttonless Brenzys autoinjector over the Enbrel autoinjector to patients (83\%; $p<0.001$, two-sided, 95\% CI [74\%, 90\%]). Almost all patients who reported past experience using an Enbrel autoinjector $(N=17)$ reported on the basis of using the two devices in the study that they would prefer to switch their device to the Brenzys autoinjector rather than continue their course of therapy using the Enbrel autoinjector $(16 / 17,94 \%, 95 \%$ CI [71\%, 100\%]).

Conclusion: On the basis of the study results, the Brenzys autoinjector was rated statistically significantly easier to use, and was overall preferred by patients and healthcare professionals with experience managing RA patients.

Funding: Merck \& Co., Inc.

Keywords: Autoinjector; Biosimilar; Enbrel; Etanercept; Human factors; Medical device; Patients; Preferences; Rheumatoid arthritis; Simulated use

\section{INTRODUCTION}

Effective biologics have been developed for management of rheumatoid arthritis (RA) using self-injection devices; however, detrimental effects of RA on manual dexterity can make self-injection an especially difficult procedure for patients to complete. Injection devices that are easier to use therefore have the potential to decrease pain and increase long-term RA therapy adherence and effectiveness. Merck \& Co., Inc. Kenilworth, NJ, USA, in collaboration with Samsung Bioepis, has been developing the Brenzys autoinjector to deliver a biosimilar of etanercept (Enbrel). The Brenzys autoinjector was designed to be easy for patients, including those with arthritis. For example, the Brenzys autoinjector was designed to have no push button requiring the thumb (the user just presses it onto skin); it allows one-handed delivery; it has a large, easy-to-grip cap; and it provides visual and auditory confirmation that the injection is complete. Compared to the Enbrel autoinjector ("MYCLIC" in Australia, "SureClick" in Canada), it has fewer steps for injection (Fig. 1).

However, to date there has been no empirical validation of the Brenzys autoinjector as a relatively easily usable option for RA patients. The current study was designed to provide data from an unbiased, counterbalanced crossover trial in which patients and healthcare professionals (HCPs) with experience managing RA (nurses and rheumatologists) injected both devices (into a pad representing skin) and reported on overall preference and ease of use (Fig. 2).

There have been several studies comparing patient perspectives on alternative injection devices. For example Berteau et al. [1], studying healthy subjects matched by demographics to be representative of RA patients, investigated patient preference for an autoinjector compared to a syringe and found an overall preference for the autoinjector. Schiff et al. [2] asked RA patients, caregivers, and HCPs to compare use of the Abatacept autoinjector to autoinjectors they had previously had experience with, with Abatacept performing favorably. Thakur et al. [3] assessed European nurses' preferences for a training version of the Benepali autoinjector compared to the Enbrel autoinjector, finding

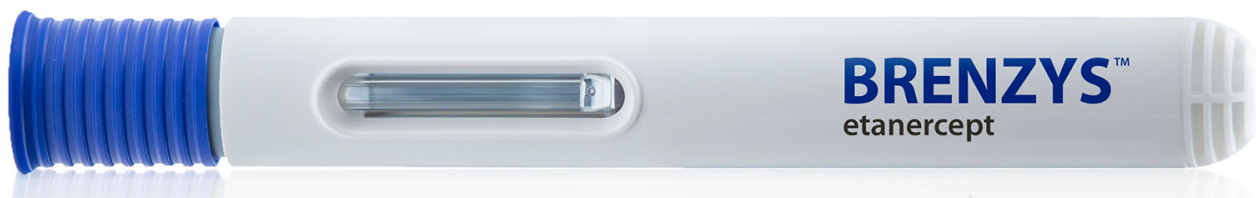

Fig. 1 BRENZYS autoinjector 
that Benepali, which is the same as the Brenzys autoinjector, was generally preferred $(86 \%$ of nurses estimated that their patients would prefer the Benepali autoinjector to the Enbrel autoinjector) (Fig. 3).

To conduct a study comparing patient and HCP preferences and perspectives on ease of use of the Brenzys autoinjector compared to Enbrel autoinjectors, we first developed and validated a questionnaire to assess autoinjector preference among RA patients and HCPs in Australia and Canada. We then enrolled 191 patients and 90 HCPs in Australia and Canada, who were blinded to the study sponsor, into a preference study. We presented each participant with both devices, in counterbalanced order between participants (approximately half of each group received the Brenzys autoinjector first, and half received the Enbrel autoinjector first). Participants received a demonstration of how to use each device, and then used each device. After they used both devices, participants were presented with a brief paper-and-pencil questionnaire. The questionnaire instructed participants to select an answer for each question. A binomial test was used to determine if the null hypothesis (i.e., there is no population-level preference for one device over another) should be rejected.

\section{METHODS}

A primary concern of this research project was in maintaining fairness in the process of testing user preferences between autoinjectors, in particular given that the study sponsor is the developer of one of the autoinjectors (Brenzys).

\section{Questionnaire Development}

Because no previous autoinjector-comparison method has been validated among our intended study populations (patients and HCPs in Australia and Canada), we first conducted an initial qualitative questionnaire development and validation phase. To design an initial questionnaire, the coauthors worked with subject matter experts at Merck \& Co., Inc., Kenilworth, NJ,
USA to outline a list of aspects of use that might differ between the Brenzys and Enbrel autoinjectors. This list was turned into a forced-choice questionnaire that was then translated into French Canadian and reviewed by linguistic experts with respect to potential word choice issues in French Canadian, Canadian English, and Australian English. A single "English" version and a single "French" version of the questionnaire resulted from this initial process.

Following initial drafting of the questionnaire, a total of 35 research participants (patients and HCPs) in Australia, English-speaking Canada, and French-speaking Canada participated in one-on-one moderated sessions. Each participant was presented with training versions of the autoinjectors and were given demonstrations of how to use the training devices. Participants then simulated use of the autoinjector training device into an injection pad. Presentation of the devices was counterbalanced between participants. Following use of the second device, participants completed the written questionnaire. After completing the questionnaire, participants were interviewed in-depth regarding their experience with and understanding of the questionnaire.

All participants in this development phase $(35 / 35,100 \%)$ indicated the same overall preference for one device over the other in the written questionnaire as during the subsequent verbal interview. Furthermore, none of the questionnaire items led more than one participant to respond differently on the written questionnaire as during the subsequent verbal interview. This process gave us confidence that the paper-and-pencil questionnaire could capture overall preference between the two autoinjectors when compared to the gold standard of an in-person discussion with a personin other words, the personal interview could be dispensed with, and the questionnaire alone could be used to learn about users' preferences.

Modifications were made to the questionnaire on the basis of a few issues encountered by participants. These changes were not validated empirically with additional study participants, but translations of changes were vetted by a team of translation experts familiar with local vernacular. 


\section{Compliance with Ethics Guidelines}

All procedures followed were in accordance with the ethical standards of the responsible committee on human experimentation (institutional and national) and with the Helsinki Declaration of 1964, as revised in 2013. Informed consent was obtained from all patients for being included in the study.

\section{Questionnaire Deployment (Testing)}

\section{Study Population}

To represent actual users, the study population in each country (Australia and Canada) included individuals from two defined user groups who met the following criteria: (1) patients with RA, including both autoinjector-naive and autoinjector-experienced; and (2) HCPs with experience managing patients with RA, including both nurses and rheumatologists. Participants provided written consent before participating in the study and were compensated for their time with an honorarium.

\section{Target Sample Size}

On the basis of estimates that a true $60 \%$ and $65 \%$ population-level preference among patients and HCPs, respectively, for one device over another would be meaningful, a minimum sample size of 188 patients and 80 HCPs was calculated to have $80 \%$ power to detect true population-level preferences for one device over the other.

\section{Study Procedures and Assessments}

Each study session employed the following procedure over the course of the sessions lasting up to $30 \mathrm{~min}$; there were no delays planned between the items listed below:

- Moderator verbally confirmed participant's consent to participate in the study, previously provided on an informed consent form.

- Moderator reviewed inclusion/exclusion criteria with participants.

- Moderator assigned participants to counterbalancing condition (which device to receive first).
- Moderator briefly showed how to use the first autoinjector.

- Participant used the first autoinjector to inject medication into an injection pad.

- Moderator briefly showed how to use the second autoinjector.

- Participant used the second autoinjector to inject medication into an injection pad.

- Participant completed paper-and-pencil questionnaire; for each item below, the questionnaire instructed the participant to circle either "The first one I used" or "The second one I used".

- Overall, which autoinjector do you prefer?

- Which one is easier to use?

- Which autoinjector was better at indicating that a full dose was actually administered?

- Which autoinjector's cap was easier to remove?

- Which autoinjector was easier to use to administer the dose?

- [Patients only]: Which autoinjector would you recommend to others?

- [HCP only]: Which autoinjector would you recommend to patients?

- [HCP only]: Which autoinjector do you think would be easier to teach patients how to use?

- [Enbrel autoinjector-experienced patients only]: If you could use either autoinjector to continue your treatment for rheumatoid arthritis, which one would you choose?

- Moderator reviewed completed questionnaire to make sure responses were legible and no responses were missing. In cases where participants would not provide an answer, saying the devices were similar, moderators encouraged participants to think more fully to find some way to differentiate. If participants would still not choose, their answer was coded as "same" and were reported but excluded from analysis of preferences.

Note that one additional questionnaire item ("Which autoinjector's label was easier to read") was initially planned but not completed because the Brenzys autoinjectors were 
unlabeled. Also note that the Enbrel MYCLIC autoinjectors available in Australia and used in the study had a dark blue plunger, while the Enbrel SureClick autoinjectors available in Canada and used in the study had a yellow plunger.

A binomial test was planned to identify statistical significance $(p<0.05)$, separately for patients and for HCPs, for each questionnaire item (except for the item intended for Enbrel autoinjector-experienced patients only, owing to the lower sample size for that item.) There was no correction for multiple comparisons because the number of planned comparisons was small, all comparisons would be presented, and questionnaire items are not independent of each other (all being related to ease of use and overall preference) making the use of a correction factor potentially misleading. To mitigate for issues related to multiple statistical comparisons, we encourage attention to the overall pattern of results rather than to results of any one statistical test. Responses broken down by additional subgroups (e.g., patients with and without limitations in hand function) are depicted without significance tests.

\section{RESULTS}

The study was conducted from May to June 2016 at research centers in Sydney, Australia; Melbourne, Australia; Brisbane, Australia; Toronto, Canada; and Montreal, Canada. A total of 191 patients reporting a diagnosis of RA and 90 HCPs with experience managing RA patients participated.

Overall, participants were more likely to indicate preferring Brenzys autoinjector than Enbrel autoinjector for all questionnaire items. Details of participant demographics and responses are shown below.

\section{Participant Demographics}

The mean age of patient participants was 52 years old and the majority of them are female which is consistent with the general RA patient demographic and included participants with $(18 \%)$ and without $(82 \%)$ autoinjector experience for RA. Most participants reported difficulty with use of their hands due to RA (92\%) (Table 1).

HCP participants included 59 nurses and 31 rheumatologists who have experience managing patients with RA (Table 2).

\section{Questionnaire Items}

Note throughout this section that sample sizes of less than 191 (patients) and 90 (HCPs) indicate participants who responded "same" or "both" on a questionnaire item.

For all survey items, patients were significantly more likely to indicate a preference for Brenzys autoinjector than Enbrel autoinjector. In the overall patient sample, the weakest preference was for cap removal $(60.0 \%$ preferring Brenzys autoinjector), and the strongest preference was in indicating which autoinjector is "easier to use" (79.1\% preferring Brenzys autoinjector) (Table 3).

HCPs were also significantly more likely to indicate a preference for Brenzys autoinjector than Enbrel autoinjector on all survey items except for cap removal. The strongest preference was in indicating which autoinjector would be easier to teach to patients $(87.8 \%$ preferring Brenzys autoinjector) (Table 4).

Four additional ad hoc two-sided binomial tests were carried out, for overall preference for Brenzys vs. Enbrel among patients and HCPs considered separately in each country. As shown in Table 5, all four of these groups were statistically more likely to indicate an overall preference (all $p<0.001$ ) for Brenzys autoinjector.

Tables 6, 7, 8, and 9 provide questionnaire responses broken down by demographic subgroups. Statistical tests were not applied.

In this study, it appears that most Canadian and Australian subgroups (i.e., by age, gender, experience with autoinjector, dexterity, etc.) were each independently more likely to prefer the Brenzys autoinjector to the Enbrel autoinjector on all survey items. These subgroup analyses demonstrated consistency in the preference for the Brenzys autoinjector across different subpopulations. 
Table 1 Patient participant demographics

\begin{tabular}{lllll}
\hline $\begin{array}{l}\text { Demographics } \\
\text { Patients with RA }\end{array}$ & $\begin{array}{l}\text { Australia } \\
(\boldsymbol{N}=\mathbf{9 0})\end{array}$ & $\begin{array}{l}\text { Canada (English) } \\
(\boldsymbol{N}=77)\end{array}$ & $\begin{array}{l}\text { Canada (French) } \\
(\boldsymbol{N}=\mathbf{2 4})\end{array}$ & $\begin{array}{l}\text { Total } \\
(\boldsymbol{N}=\mathbf{1 9 1})\end{array}$ \\
\hline $\begin{array}{l}\text { Gender } \\
\text { Female }\end{array}$ & $59(65.6 \%)$ & $55(71.4 \%)$ & $15(62.5 \%)$ & $129(67.5 \%)$ \\
Average age (years) & 52.5 & 51.7 & 50.9 & 52 \\
Age (years) & $15(16.7 \%)$ & $14(18.2 \%)$ & $10(41.7 \%)$ & $39(20.4 \%)$ \\
$18-39$ & $18(20.0 \%)$ & $23(29.9 \%)$ & $0(0.0 \%)$ & $41(21.5 \%)$ \\
$40-49$ & $25(27.8 \%)$ & $18(23.4 \%)$ & $5(20.8 \%)$ & $48(25.1 \%)$ \\
$50-59$ & $25(27.8 \%)$ & $15(19.5 \%)$ & $6(25.0 \%)$ & $46(24.1 \%)$ \\
$60-69$ & $7(7.8 \%)$ & $7(9.1 \%)$ & $3(12.5 \%)$ & $17(8.9 \%)$ \\
$70+$ & $74(82.2 \%)$ & $64(83.1 \%)$ & $19(79.2 \%)$ & $157(82.2 \%)$ \\
Autoinjector experience for rheumatoid arthritis & & & $175(91.6 \%)$ \\
Naive & $84(93.3 \%)$ & $70(90.9 \%)$ & $19(79.2 \%)$ & $177(92.7 \%)$ \\
Injection experience other than rheumatoid arthritis & $69(89.6 \%)$ & & \\
Naive & $89(98.9 \%)$ & &
\end{tabular}

a Participants were asked the following question, and those who indicated anything other than "none of the above" were considered to have difficulty with dexterity:

With regard to your hands, wrists, and fingers, which if any of the following do you have on a regular basis?

(a) Significant hand weakness making it difficult to hold heavy objects

(b) Significant pain making it difficult to move your hands or grasp objects

(c) Limited dexterity making it difficult to pick up, hold, and/or manipulate small objects (i.e., coins, buttons, pencils, etc.)

(d) Limited range of motion making it difficult to fully open or close your hand or rotate your wrists

(e) Deformities in the joints of your hands causing your fingers/thumbs to twist

(f) None of the above

\section{DISCUSSION}

The results from this study suggest that RA patients and HCPs would significantly prefer the Brenzys autoinjector over the Enbrel autoinjector when choosing their injection device for their RA treatment. In addition, they would also be more likely to recommend the Brenzys autoinjector to their peers and patients. Subgroup analyses also indicate that a majority of English-Canadian, French-Canadian, and Australian RA patients and HCPs have an overall preference for the Brenzys autoinjector including patients with and without limitations in hand function, patients with and without previous autoinjector experience, male and female, different age groups, nurses, and rheumatologists.

Almost all RA patient participants who had past experience with the Enbrel autoinjector indicated that they would prefer to use the Brenzys autoinjector. Participants were selected to be representative of real-world users, leading to the conclusion that RA patients and HCPs with experience managing RA would prefer and/or recommend the Brenzys autoinjector over the Enbrel autoinjector. 
Table 2 HCP participant demographics

\begin{tabular}{lllll}
\hline $\begin{array}{l}\text { Demographics } \\
\text { HCPs }\end{array}$ & $\begin{array}{l}\text { Australia } \\
(\boldsymbol{n}=\mathbf{3 9})\end{array}$ & $\begin{array}{l}\text { Canada (English) } \\
(\boldsymbol{n}=\mathbf{3 6})\end{array}$ & $\begin{array}{l}\text { Canada (French) } \\
(\boldsymbol{n}=\mathbf{1 5})\end{array}$ & $\begin{array}{l}\text { Total } \\
(\boldsymbol{n}=\mathbf{9 0})\end{array}$ \\
\hline Gender & $30(76.9 \%)$ & $25(69.4 \%)$ & $13(86.7 \%)$ & $68(75.6 \%)$ \\
Female & 46.0 & 52.3 & 46.7 & 48.7 \\
Average age (years) & & & \\
Age, years (avg. age) & $11(28.2 \%)$ & $3(8.3 \%)$ & $6(40.0 \%)$ & $20(22.2 \%)$ \\
$18-39$ & $12(30.8 \%)$ & $10(27.8 \%)$ & $3(20.0 \%)$ & $25(27.8 \%)$ \\
$40-49$ & $15(38.5 \%)$ & $15(41.7 \%)$ & $2(13.3 \%)$ & $32(35.6 \%)$ \\
$50-59$ & $1(2.6 \%)$ & $6(16.7 \%)$ & $3(20.0 \%)$ & $10(11.1 \%)$ \\
$60-69$ & $0(0 \%)$ & $2(5.6 \%)$ & $1(6.7 \%)$ & $3(3.3 \%)$ \\
$70+$ & & & $12(80.0 \%)$ & $59(65.6 \%)$ \\
Position & $30(76.9 \%)$ & $17(47.2 \%)$ & $3(20.0 \%)$ & $31(34.4 \%)$ \\
Nurse & $9(23.1 \%)$ & $19(52.8 \%)$ & & \\
Rheumatologist & &
\end{tabular}

Table 3 Patient questionnaire results

\begin{tabular}{|c|c|c|c|c|}
\hline & \multicolumn{4}{|c|}{ Patients } \\
\hline & $\bar{N}$ & Prefer Brenzys & 95\% CI & $p$ value \\
\hline Overall, which autoinjector do you prefer? & 191 & $142(74.3 \%)$ & {$[67.5 \%, 80.3 \%]$} & $<0.001$ \\
\hline Which autoinjector is easier to use? & 191 & $151(79.1 \%)$ & {$[72.5 \%, 84.6 \%]$} & $<0.001$ \\
\hline $\begin{array}{l}\text { Which autoinjector was better at indicating that a full } \\
\text { dose was actually administered? }\end{array}$ & 188 & $119(63.3 \%)$ & {$[55.9 \%, 70.1 \%]$} & $<0.001$ \\
\hline Which autoinjector's cap was easier to remove? & 190 & $114(60.0 \%)$ & {$[52.5 \%, 67.0 \%]$} & $<0.05$ \\
\hline Which autoinjector was easier to use to administer the dose? & 191 & $150(78.5 \%)$ & {$[72.0 \%, 84.1 \%]$} & $<0.001$ \\
\hline Which autoinjector would you recommend to others? & 191 & $141(73.8 \%)$ & {$[66.9 \%, 79.9 \%]$} & $<0.001$ \\
\hline $\begin{array}{l}\text { [Enbrel autoinjector-experienced only]: If you could use } \\
\text { either autoinjector to continue your treatment for rheumatoid } \\
\text { arthritis, which one would you choose? }\end{array}$ & 17 & $16(94.1 \%)$ & {$[71.3 \%, 99.8 \%]$} & $\mathrm{N} / \mathrm{A}^{\mathrm{a}}$ \\
\hline
\end{tabular}

${ }^{a}$ Not tested because of sample size

In the questionnaire development and validation phase of this study, some patient and HCP participants reported during interviews that injecting with the Enbrel autoinjector could be painful for patients with limitations in hand function because of the button press 


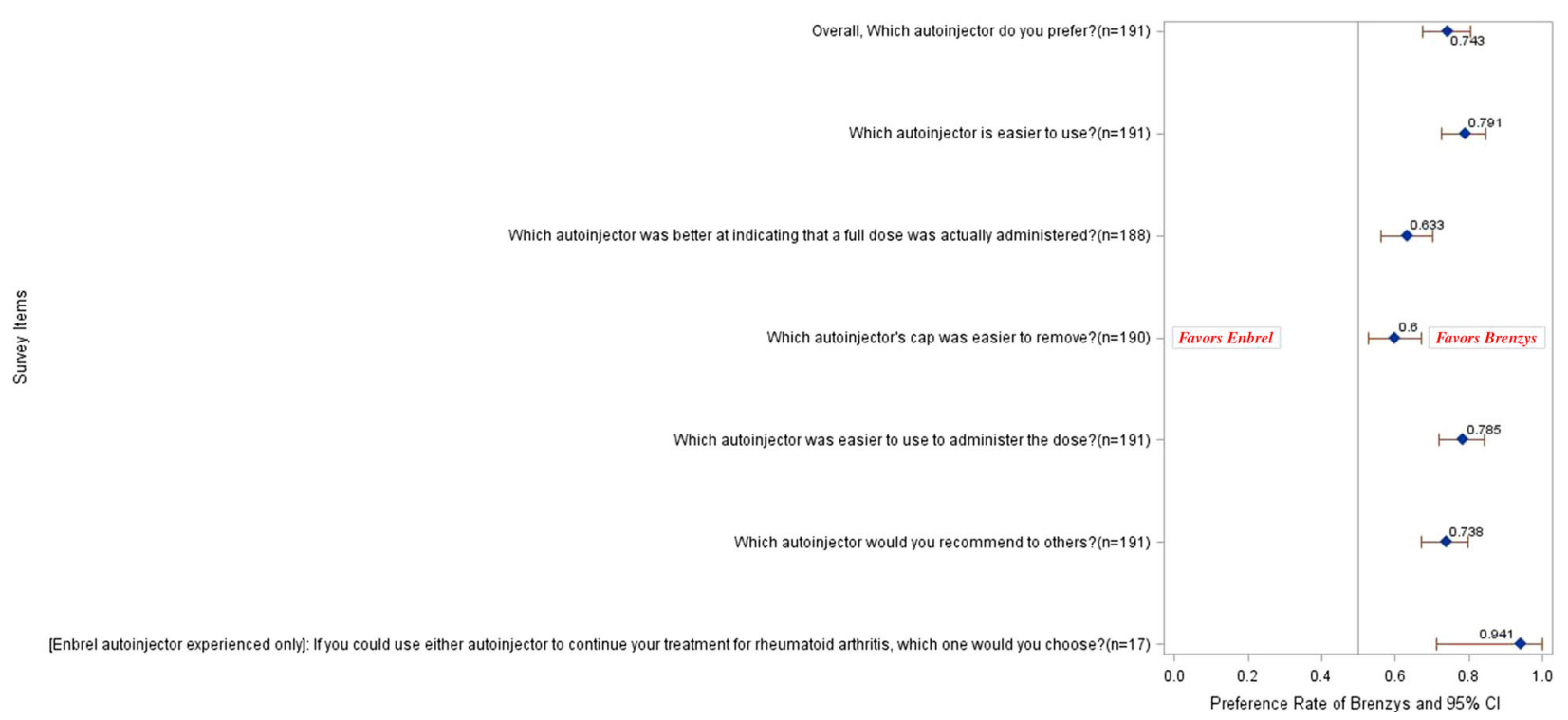

Fig. 2 Forest plot of patient preference

Table 4 HCP questionnaire results

\begin{tabular}{|c|c|c|c|c|}
\hline & \multicolumn{4}{|c|}{ HCP } \\
\hline & $\bar{N}$ & $\begin{array}{l}\text { Prefer } \\
\text { Brenzys }\end{array}$ & $95 \% \mathrm{CI}$ & $p$ value \\
\hline Overall, which autoinjector do you prefer? & 90 & $72(80.0 \%)$ & $\begin{array}{l}{[70.2 \%} \\
87.6 \%]\end{array}$ & $<0.001$ \\
\hline Which autoinjector is easier to use? & 90 & $77(85.6 \%)$ & $\begin{array}{l}{[76.5 \%} \\
92.0 \%]\end{array}$ & $<0.001$ \\
\hline $\begin{array}{l}\text { Which autoinjector was better at indicating that a full dose was } \\
\text { actually administered? }\end{array}$ & 87 & $67(77.0 \%)$ & $\begin{array}{l}{[66.7 \%} \\
85.3 \%]\end{array}$ & $<0.001$ \\
\hline Which autoinjector's cap was easier to remove? & 87 & $48(55.2 \%)$ & $\begin{array}{l}{[44.1 \%} \\
65.8 \%]\end{array}$ & $\begin{array}{l}\text { Not significant } \\
\qquad(p=0.053)\end{array}$ \\
\hline Which autoinjector was easier to use to administer the dose? & 90 & $76(84.4 \%)$ & $\begin{array}{l}{[75.2 \%} \\
91.2 \%]\end{array}$ & $<0.001$ \\
\hline Which autoinjector would you recommend to patients? & 89 & $74(83.1 \%)$ & $\begin{array}{l}{[73.7 \%} \\
90.2 \%]\end{array}$ & $<0.001$ \\
\hline $\begin{array}{l}\text { Which autoinjector do you think would be easier to teach patients } \\
\text { how to use? }\end{array}$ & 90 & $79(87.8 \%)$ & $\begin{array}{l}{[79.1 \%} \\
93.7 \%]\end{array}$ & $<0.001$ \\
\hline
\end{tabular}

which requires thumb use, and commented that the buttonless Brenzys autoinjector would work better for patients with RA who might have limitations in hand function. Thakur et al. [3] similarly found preferences for a new etanercept autoinjector based on a buttonless 


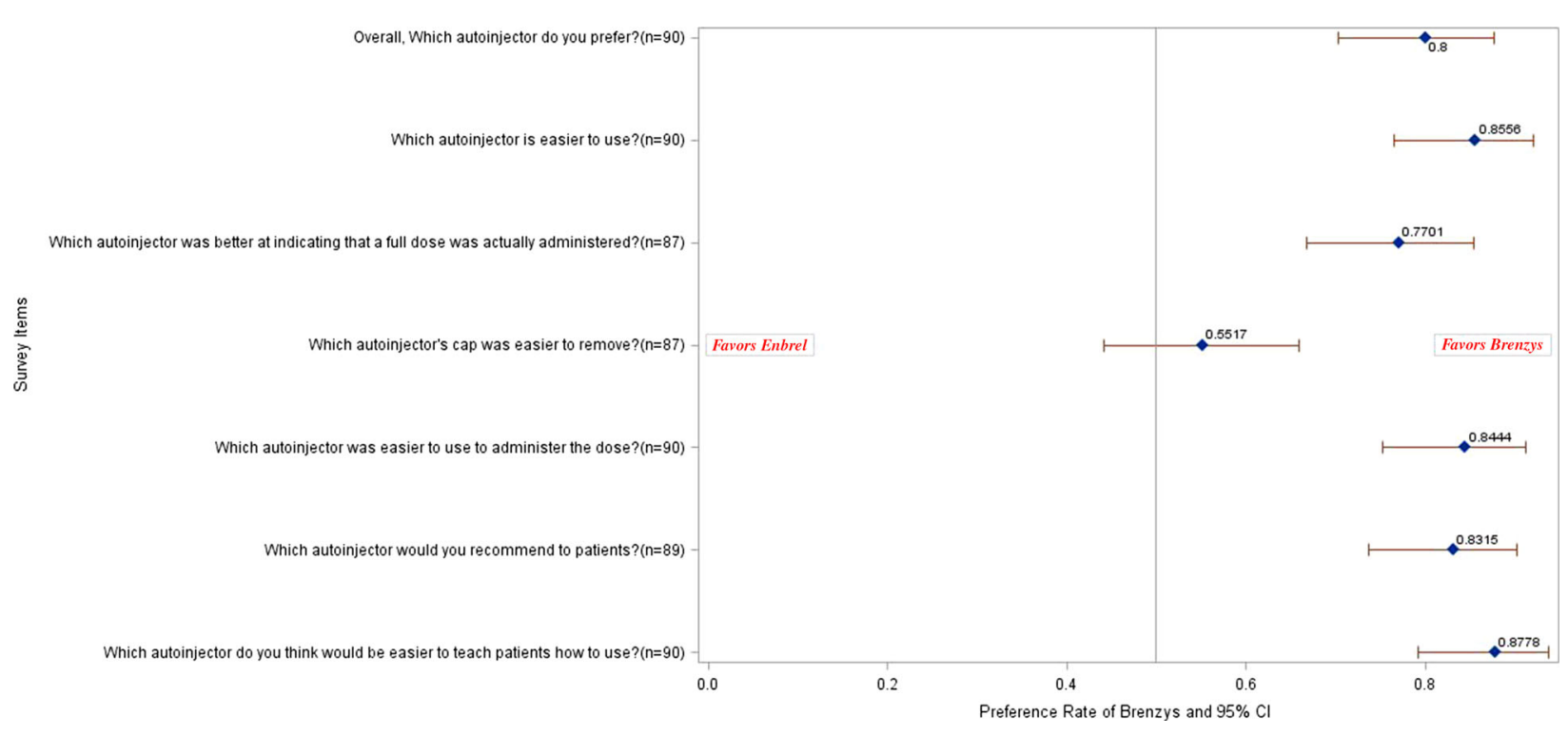

Fig. 3 Forest plot of HCP preference

Table 5 Overall preference (questionnaire item 1), patients and HCPs in Australia and Canada

\begin{tabular}{|c|c|c|c|c|c|c|}
\hline & \multicolumn{3}{|c|}{ RA patients } & \multicolumn{3}{|c|}{ HCPs (nurses and rheumatologists) } \\
\hline & $\bar{N}$ & $\%$ preferring Brenzys overall & $p$ value & $\bar{N}$ & \% preferring Brenzys overall & $p$ value \\
\hline Australia & 90 & 83.3 & $<0.001$ & 39 & 85.0 & $<0.001$ \\
\hline Canada & 101 & 66.3 & $<0.001$ & 51 & 76.5 & $<0.001$ \\
\hline
\end{tabular}

design. In addition to confirming relative ease of use to perform the actual injection with the Brenzys autoinjector, the current study also found that patients prefer the Brenzys cap removal (cap removal also requiring manual dexterity). The Brenzys dose feedback mechanism (plunger visible in a window) indicating a complete dose was also overall preferred to Enbrel's.

Nurses and rheumatologists in the current study indicated that they would recommend the Brenzys rather than Enbrel autoinjector to patients, and also indicated that they thought the Brenzys autoinjector would be easier to teach to patients than to the Enbrel autoinjector. An autoinjector that is simpler to learn and easier to use has the potential to reduce time taken in training sessions and has the potential to improve long-term adherence to, and thereby effectiveness of, a course of therapy. Further research would be needed to assess actual impacts of such a preferred device on adherence, safety, effectiveness, and healthcare costs.

\section{Limitations}

Participants injected into an injection pad, rather than into themselves, and only once 


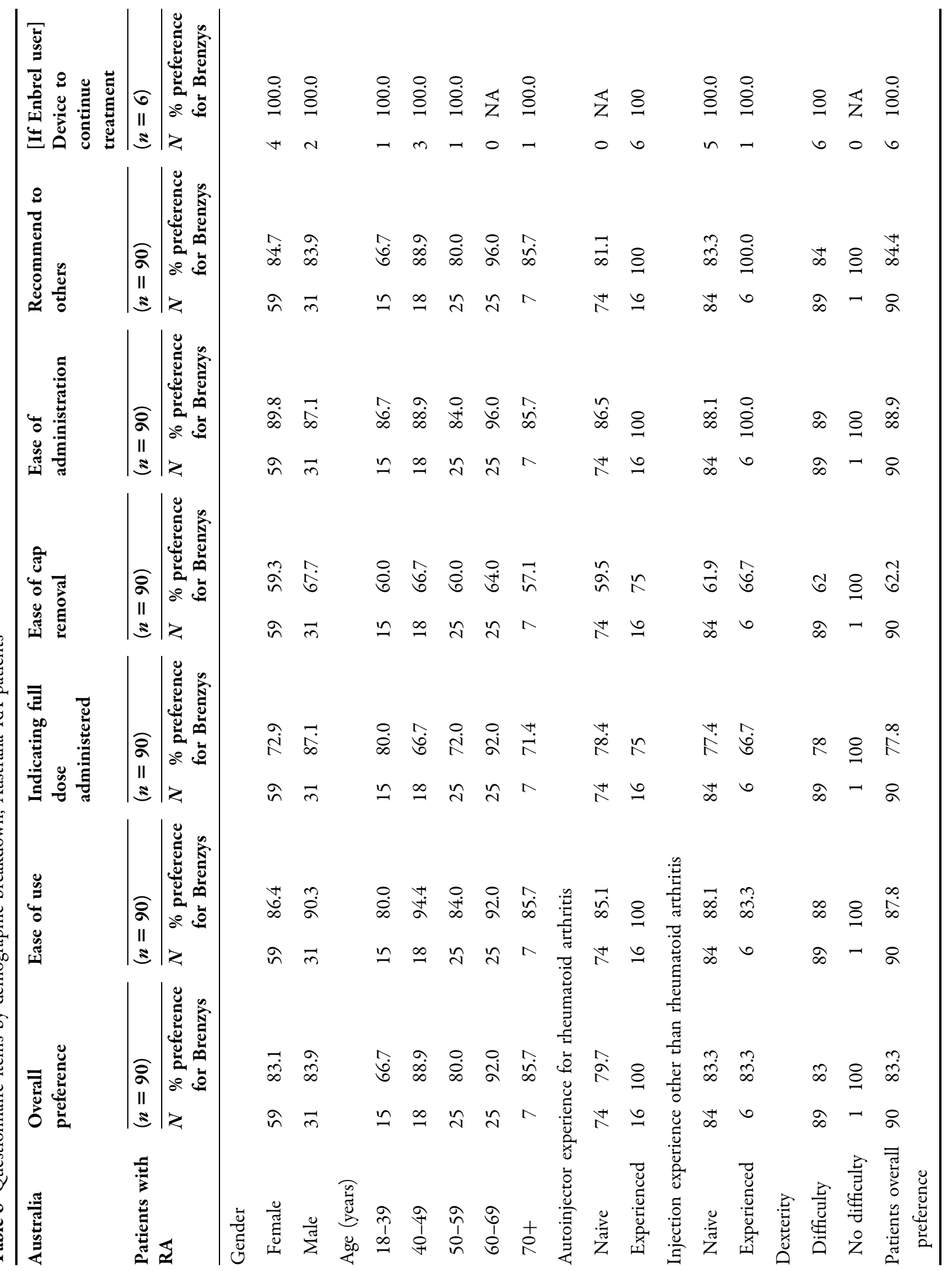




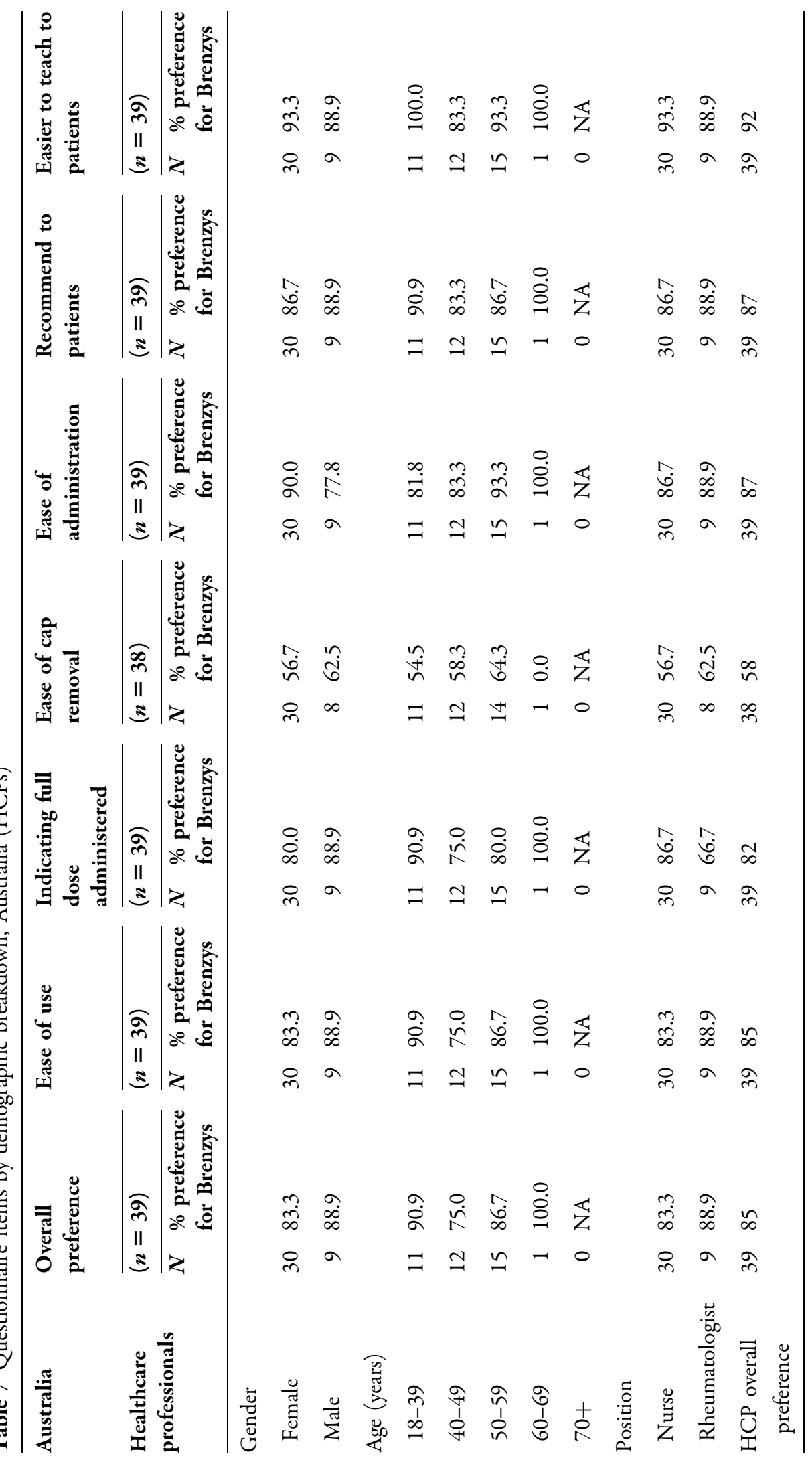




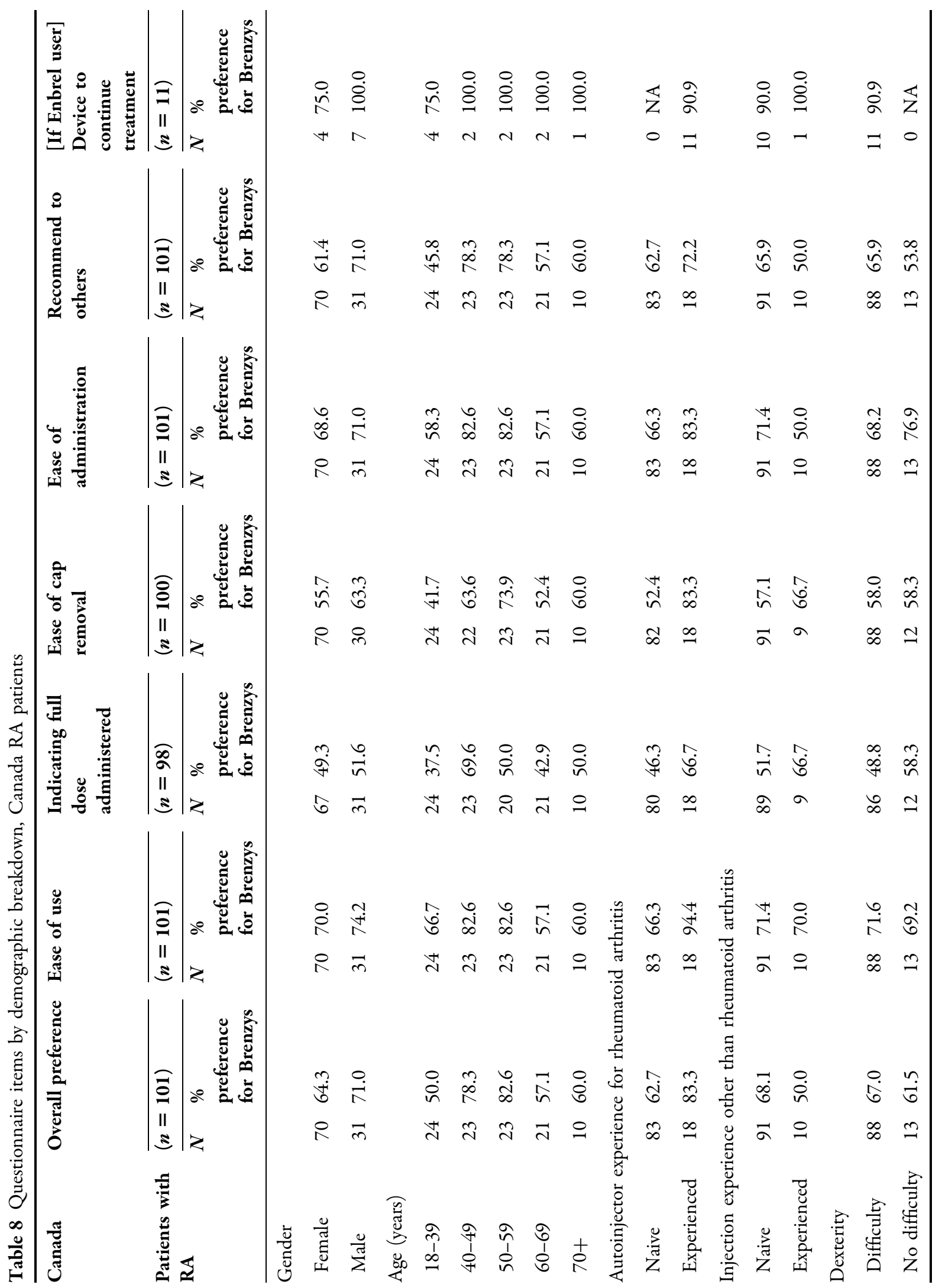




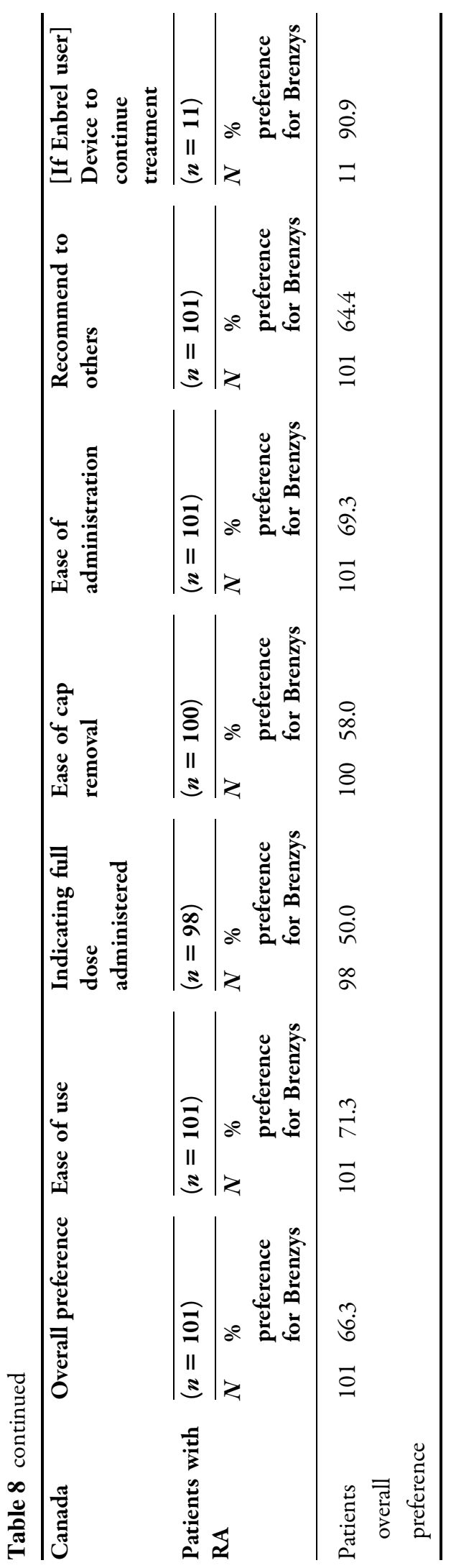

instead of repeatedly over the course of therapy. It is possible that long-term use or use into actual skin may yield different patterns of preferences, although there is no specific reason that this would be the case.

The Brenzys autoinjector was preferred by participants, but it was not in the scope of the current study to record and analyze use errors, so the preference for ease of use of the Brenzys autoinjector or the Enbrel autoinjector is not equivalent to an assessment of safe and effective use of either device.

Patient dexterity and difficulties with dexterity were self-assessed, but not independently verified.

The study was conducted in Australia and Canada. Users in other locations might have different impressions of the autoinjectors, although there is no specific reason we know of that this would be the case.

\section{CONCLUSIONS}

RA patients and nurses and rheumatologists with experience managing RA patients indicated an overall preference for using the Brenzys autoinjector, rather than the Enbrel autoinjector, after using both devices. The preference extended to various aspects of use, teaching, and recommendations participants would make to others. Both HCPs and patients were more likely to indicate that they would recommend to others the Brenzys autoinjector rather than the Enbrel autoinjector. Owing to the design of the study, we expect that readers can use this data to understand the experiences of real-world users using devices. The study allows us to infer that real-life users, including RA patients with limitations in hand function, would have a better injection experience were they to use the Brenzys autoinjector rather than the Enbrel autoinjector. DiMatteo [4] and Mohr et al. [5] found that difficulty injecting can lead to increased and unnecessary pain, lack of adherence, and increased overall treatment costs. In light of the importance of ease of use and preference by patients and providers, healthcare stakeholders (patients, lay caregivers, nurses, doctors, payers, policy makers, etc.) can use 


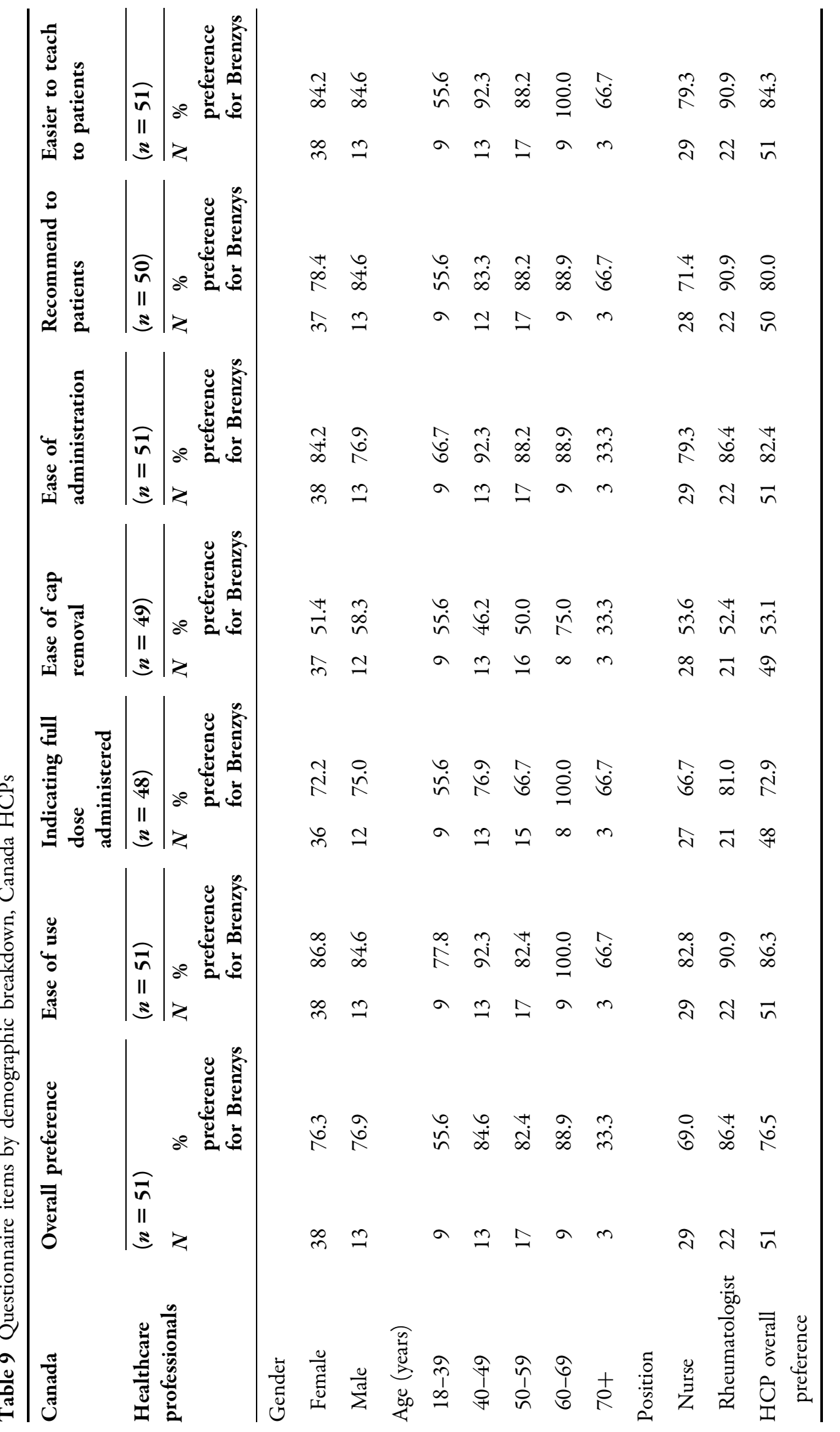


evidence from this study to assist their decision-making when selecting Brenzys or Enbrel for RA management.

\section{ACKNOWLEDGEMENTS}

This study was commissioned by Merck \& Co., Inc., Kenilworth, NJ, USA. Article processing charges were funded by Merck \& Co. Thanks to Renna Patel, Jinan Liu, and Girish Prajapati. All authors had full access to all of the data in this study and take complete responsibility for the integrity of the data and accuracy of the data analysis. All named authors meet the International Committee of Medical Journal Editors (ICMJE) criteria for authorship for this manuscript, take responsibility for the integrity of the work as a whole, and have given final approval for the version to be published. Merck and Co, Inc. ("Merck") hired Core Human Factors, Inc. ("Core"), an independent research group, to design, conduct, and write up a study comparing use of Brenzys autoinjector to Enbrel autoinjector in Australia and Canada. Merck had a hypothesis that Brenzys was easier to use than Enbrel owing to its one-press form factor. Merck commented on and approved research designs throughout the research process. The resulting study design compared the two autoinjectors in an unbiased way on the factors hypothesized (described in the paper as hypotheses) to favor Brenzys. Researchers from Core moderated sessions or independently managed non-English-speaking moderators to interview study participants, gathered the data, and conducted the analyses. Other co-authors include employees either at Merck or at Core, as listed in affiliations. The results seem to the authors to be valid and to represent the contribution to developments in therapy as described in the paper including the conclusions.

Disclosures. Marc Egeth is an employee of Core Human Factors Inc, an independent research company engaged by (paid by) Merck and Co. to design and conduct the study. Jennifer Soosaar is an employee of Core Human Factors Inc, an independent research company engaged by (paid by) Merck and Co. to design and conduct the study. Peter Nash is a prior paid consultant of Merck \& Co., not paid with respect to the current research. Denis Choquette is a prior paid consultant of Merck \& Co., not paid with respect to the current research. Ricardo Infante is an employee of Merck \& Co. Dena Rosen Ramey is an employee of Merck \& Co. Sevag Sahakian is an employee of Merck \& Co. Angela Lai is an employee of Merck \& Co. Jin Ju Kim is an employee of Merck \& Co. David $\mathrm{Wu}$ is an employee of Merck \& Co.

Compliance with Ethics Guidelines. All procedures followed were in accordance with the ethical standards of the responsible committee on human experimentation (institutional and national) and with the Helsinki Declaration of 1964, as revised in 2013. Informed consent was obtained from all patients for being included in the study.

Data Availability. The datasets analyzed during the current study are available from the corresponding author on reasonable request.

Open Access. This article is distributed under the terms of the Creative Commons Attribution-NonCommercial 4.0 International License (http://creativecommons.org/licenses/ by-nc/4.0/), which permits any noncommercial use, distribution, and reproduction in any medium, provided you give appropriate credit to the original author(s) and the source, provide a link to the Creative Commons license, and indicate if changes were made.

\section{REFERENCES}

1. Berteau C, Schwarzenback F, Donazzolo Y, et al. Evaluation of performance, safety, subject acceptance, and compliance of a disposable autoinjector for subcutaneous injections in healthy volunteers. Patient Pref Adher. 2010;4:379-88.

2. Schiff M, Koo J, Jin E, Schiller E, Day A. Usability and acceptability of the Abatercept pre-filled autoinjector for the subcutaneous treatment of rheumatoid arthritis. Adv Ther. 2016;33:199-213. 
3. Thakur K, Biberger A, Handrich A, Rezk MF. Perceptions and preferences of two etanercept autoinjector for rheumatoid arthritis: a new European Union-approved etanercept biosimilar (Benepali) versus etanercept (Enbrel)-findings from a nurse questionnaire in Europe. Rheumatol Ther. 2016;3(1):77-89.

4. DiMatteo MR. Variations in patient's adherence to medical recommendation: a quantitative review of 50 years of research. Med Care. 2004;42:200-9.
5. Mohr DC, Cox D, Epstein L, Boudewyn A. Teaching patients to self-inject: pilot study of a treatment for injection anxiety and phobia in multiple sclerosis patients prescribed injectable medication. J Behav Ther Exp Psychiatry. 2002;33:39-47. 